\title{
Flavor Physics and Fine-Tuning in Theory Space
}

\author{
R. Sekhar Chivukula ${ }^{1}$, Nick Evans ${ }^{3}$, and Elizabeth H. Simmons ${ }^{1,2 *}$ \\ ${ }^{1}$ Department of Physics, Boston University, \\ 590 Commonwealth Ave., Boston MA 02215 \\ ${ }^{2}$ Department of Physics, Harvard University, \\ Cambridge, MA, 02138 \\ ${ }^{3}$ Dept. of Physics, University of Southampton, \\ Highfield, Southampton, SO17 1BJ, U.K.
}

April 26, 2002

\begin{abstract}
Recently a new class of composite Higgs models have been developed which give rise to naturally light Higgs bosons without supersymmetry. Based on the chiral symmetries of "theory space," involving replicated gauge groups and appropriate gauge symmetry breaking patterns, these models allow the scale of the underlying strong dynamics giving rise to the composite particles to be as large as of order $10 \mathrm{TeV}$, without any fine tuning to prevent large corrections to Higgs boson mass(es) of order $100 \mathrm{GeV}$. In this note we show that the size of flavor violating interactions arising generically from underlying flavor dynamics constrain the scale of the Higgs boson compositeness to be greater than of order $75 \mathrm{TeV}$, implying that significant fine-tuning is required. Without fine-tuning, the low-energy structure of the composite Higgs model alone is not sufficient to eliminate potential problems with flavor-changing neutral currents or excessive CP violation; solving those problems requires additional information or assumptions about the symmetries of the underlying flavor or strong dynamics. We also consider the weaker, but more model-independent, bounds which arise from limits on weak isospin violation.
\end{abstract}

*e-mail addresses sekhar@bu.edu, evans@phys.soton.ac.uk, simmons@bu.edu 


\section{Introduction}

Recently a new class [1, 2] of composite Higgs models [3] has been developed which give rise to naturally light Higgs bosons without supersymmetry. Inspired by discretized versions of higher-dimensional gauge theory [4, 5], these models are based on the chiral symmetries of "theory space" 四] The models involve replicated gauge groups and corresponding gauge symmetry breaking patterns. They allow the scale $(\Lambda)$ of the underlying strong dynamics giving rise to the composite particles to be as large as $10 \mathrm{TeV}$, without causing large corrections to the Higgs boson mass(es) of order $100 \mathrm{GeV}$.

Various possibilities exist for the underlying physics (the "high-energy completion") which gives rise to the chiral-symmetry breaking pattern required, and produces the "pion" which becomes the composite Higgs. However, regardless of the precise nature of the underlying strongly-interacting physics, there must be flavor dynamics at a scale of order $\Lambda$ or greater that gives rise to the different Yukawa couplings of the Higgs boson to ordinary fermions. As in extended technicolor theories [6, [], if this flavor dynamics arises from gauge-interactions it will generically cause flavor-changing neutral currents [7].

In this note we review and update the lower bound on $\Lambda$ arising from the experimental constraints on extra contributions to the neutral meson mass differences [8]. We find that in composite Higgs models the size of flavor-violating interactions arising from the high-energy theory constrain the scale $\Lambda$ to be greater than of order $75 \mathrm{TeV}$. We then consider the "theory space" models, argue why this flavor bound applies to such models, and review the upper limit on $\Lambda$ of order $10 \mathrm{TeV}$ necessary to avoid fine-tuning [1, 2]. Raising the scale $\Lambda$ to $75 \mathrm{TeV}$ to be consistent with the flavor bounds mentioned above, then, necessitates fine tuning of order $2 \%$. We compare these bounds to those arising from limits on the amount of $\mathrm{CP}$ violation and isospin violation in the composite Higgs theory.

The implication of our findings is that the low-energy structure of the composite Higgs model alone is not sufficient to eliminate potential problems with flavor-changing neutral current or excessive $\mathrm{CP}$ violation; solving those problems requires additional information or assumptions about the symmetries of the underlying strong dynamicst.

\section{Flavor and Composite Higgs Bosons}

We begin by considering what the observed masses of the ordinary fermions imply about the underlying flavor physics. Providing the different masses of the fermions requires flavor physics (analogous to extended-technicolor interactions (ETC) [6, [])

\footnotetext{
${ }^{1}$ See also [9], which emphasizes that the properties of the underlying strong-dynamics may affect the details of the low-energy phenomenology.

${ }^{2}$ This section reviews and updates material from 8 .
} 
which couples the left-handed quark doublets $\psi_{L}$ and right-handed singlets $q_{R}$ to the strongly-interacting constituents of the composite Higgs doublet. At low energies, these interactions produce the quark Yukawa couplings.

To estimate the sizes of various effects of the underlying physics, we rely on dimensional analysis [10]. As noted by Georgi [11], a theory with light scalar particles belonging to a single symmetry-group representation depends on two parameters: $\Lambda$, the scale of the underlying physics, and $f$ (the analog of $f_{\pi}$ in QCD), which measures the amplitude for producing the scalar particles from the vacuum. Our estimates of the sizes of the low-energy effects of the underlying physics will depend on the ratio $\kappa \equiv \Lambda / f$, which determines the sizes of coupling constants in the low-energy theory. Naive dimensional analysis corresponds to $\kappa=4 \pi[10$.

Assuming that these new flavor interactions are gauge interactions with gauge coupling $g$ and gauge boson mass $M$, dimensional analysis [10] allows us to estimate that the size of the resulting Yukawa coupling is [3] of order $\left(g^{2} / M^{2}\right)\left(\Lambda^{2} / \kappa\right)$, i.e.

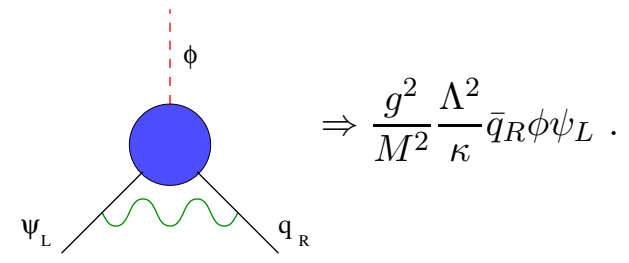

In order to give rise to a quark mass $m_{q}$, the Yukawa coupling must be equal to

$$
\frac{\sqrt{2} m_{q}}{v}
$$

where $v \approx 246 \mathrm{GeV}$. This implies

$$
\Lambda \approx \frac{M}{g} \sqrt{\sqrt{2} \kappa \frac{m_{q}}{v}} .
$$

Thus, if we set a lower limit on $M / g$ from low-energy flavor physics, eqn.(3) will give a lower bound on $\Lambda$.

The high-energy flavor physics responsible for the generation of the Yukawa couplings must distinguish between different flavors so as to give rise to the different masses of the corresponding fermions. In addition, the flavor physics will give rise to flavor-specific couplings among ordinary fermions [6, 7]. These will generically give rise to flavor-changing neutral currents (as previously noted in [7] for the case of ETC theories) that affect Kaon, $D$-meson, and $B$-meson physics.

Consider the interactions responsible for the $c$-quark mass. Through Cabibbo mixing, these interactions must couple to the $u$-quark as well. Neglecting mixing with the top-quark, this will generally give rise to the interactions

$$
\mathcal{L}_{e f f}=-\left(\cos \theta_{L}^{c} \sin \theta_{L}^{c}\right)^{2} \frac{g^{2}}{M^{2}}\left(\bar{c}_{L} \gamma^{\mu} u_{L}\right)\left(\bar{c}_{L} \gamma_{\mu} u_{L}\right)
$$




$$
\begin{aligned}
& -\left(\cos \theta_{R}^{c} \sin \theta_{R}^{c}\right)^{2} \frac{g^{2}}{M^{2}}\left(\bar{c}_{R} \gamma^{\mu} u_{R}\right)\left(\bar{c}_{R} \gamma_{\mu} u_{R}\right) \\
& -2 \cos \theta_{L}^{c} \sin \theta_{L}^{c} \cos \theta_{R}^{c} \sin \theta_{R}^{c} \frac{g^{2}}{M^{2}}\left(\bar{c}_{L} \gamma^{\mu} u_{L}\right)\left(\bar{c}_{R} \gamma_{\mu} u_{R}\right),
\end{aligned}
$$

where the coupling $g$ and mass $M$ are of the same order as those in the interactions which ultimately give rise to the $c$-quark Yukawa coupling in eqn. (11), and the angles $\theta_{L}^{c}$ and $\theta_{R}^{c}$ represent the relation between the gauge eigenstates and the mass eigenstates. The operators in eqn. (4) will clearly affect neutral D-meson physics. Similarly, the interactions responsible for other quarks' masses will give rise to operators that contribute to mixing and decays of the corresponding mesons.

The color-singlet products of currents in eqn. (44) will contribute directly to $D$ meson mixing. In the vacuum-insertion approximation, the purely left-handed or right-handed current-current operators yield

$$
\left(\frac{M}{g}\right)_{\mathrm{LL}, \mathrm{RR}} \gtrsim f_{D}\left(\frac{2 m_{D} B_{D}}{3 \Delta m_{D}}\right)^{1 / 2} \cos \theta_{L, R}^{c} \sin \theta_{L, R}^{c} \approx 225 \mathrm{TeV},
$$

where we have used the limit on the neutral $D$-meson mass difference, $\Delta m_{D} \lesssim$ $4.6 \times 10^{-11} \mathrm{MeV}$ [12], and $f_{D} \sqrt{B_{D}}=0.2 \mathrm{GeV}$ [13], $\theta_{L, R}^{c} \approx \theta_{C}$. The bound on the scale of the underlying strongly-interacting dynamics follows from eqn. (3):

$$
\Lambda \gtrsim 21 \mathrm{TeV} \sqrt{\kappa\left(\frac{m_{c}}{1.5 \mathrm{GeV}}\right)}
$$

so that $\Lambda \gtrsim 75 \mathrm{TeV}$ for $\kappa \approx 4 \pi$.

The $\Delta C=2$, LR product of color-singlet currents gives a weaker bound than eqn. (6), but the LR product of color-octet currents,

$$
\mathcal{L}_{e f f}=-2 \cos \theta_{L}^{c} \sin \theta_{L}^{c} \cos \theta_{R}^{c} \sin \theta_{R}^{c} \frac{g^{2}}{M^{2}}\left(\bar{c}_{L} \gamma^{\mu} T^{a} u_{L}\right)\left(\bar{c}_{R} \gamma_{\mu} T^{a} u_{R}\right)
$$

where $T^{a}$ are the generators of $S U(3)_{C}$, gives a stronger bound:

$$
\begin{aligned}
\left(\frac{M}{g}\right)_{\mathrm{LR}} & \gtrsim \frac{4 f_{D}}{3\left(m_{c}+m_{u}\right)}\left(\frac{m_{D}^{3} B_{D}^{\prime}}{\Delta m_{D}}\right)^{1 / 2}\left(2 \cos \theta_{L}^{c} \sin \theta_{L}^{c} \cos \theta_{R}^{c} \sin \theta_{R}^{c}\right)^{1 / 2} \\
& \approx 590 \mathrm{TeV}\left(\frac{1.5 \mathrm{GeV}}{m_{c}}\right)
\end{aligned}
$$

corresponding to

$$
\Lambda \gtrsim 53 \mathrm{TeV} \sqrt{\kappa\left(\frac{1.5 \mathrm{GeV}}{m_{c}}\right)}
$$


There are also contributions to $K$-meson mixing from the color-singlet and coloroctet products of currents analogous to those in eqns. (14) and (7). The lower bound on $\Lambda$ derived from the measured value of the $K_{L} K_{S}$ mass difference [8]

$$
\Lambda \gtrsim 6.8 \mathrm{TeV} \sqrt{\kappa\left(\frac{m_{s}}{200 \mathrm{MeV}}\right)} .
$$

is weaker than (16) because the $s$-quark is lighter than the $c$-quark, while the $d-s$ and $u-c$ mixings are expected to be of comparable size [8]. However, in the absence of additional superweak interactions to give rise to CP-violation in $K$-mixing $(\varepsilon)$, the flavor interactions responsible for the $s$-quark Yukawa couplings must violate $\mathrm{CP}$ at some level. In this case the the bounds on the scale $\Lambda$ are much stronger. Recalling that

$$
\operatorname{Re} \varepsilon \approx \frac{\operatorname{ImM}_{12}}{2 \Delta M} \lesssim 1.65 \times 10^{-3},
$$

and assuming that there are phases of order 1 in the $\Delta S=2$ operators analogous to those shown in eqn. (4), we find the bound

$$
\Lambda \gtrsim 120 \mathrm{TeV} \sqrt{\kappa\left(\frac{m_{s}}{200 \mathrm{MeV}}\right)} .
$$

\section{Composite Higgs Bosons from Theory Space}

A set of "theory space" composite Higgs models [1, 2] is illustrated in Figure 1, using "moose" or "quiver" notation [14]. In this diagram, each site except $(1,1)$ represents a gauged $S U(3)$ group, while the links represent non-linear sigma fields transforming as $(3, \overline{3})$ 's under the adjacent groups:

$$
U_{i j} \rightarrow W_{i j} U_{i j} W_{i j+1}^{\dagger}, \quad V_{i j} \rightarrow W_{i j} V_{i j} W_{i+1 j}^{\dagger} .
$$

The "toroidal" geometry of theory space implies that the indices $i, j$ are periodic $\bmod N$. At the site $(1,1)$, only the $S U(2) \times U(1)$ subgroup of an $S U(3)$ global symmetry is gauged. The kinetic energy terms in the Lagrangian then read

$$
\mathcal{L}_{k i n}=-\sum_{i j} \frac{1}{2 g_{i j}^{2}} \operatorname{Tr} F_{i j}^{2}+\frac{f^{2}}{4} \sum_{i j} \operatorname{Tr}\left|D^{\mu} U_{i j}\right|^{2}+\frac{f^{2}}{4} \sum_{i j} \operatorname{Tr}\left|D^{\mu} V_{i j}\right|^{2},
$$

where $g_{i j}$ are the gauge couplings and $f$ is the "pion-decay constant" of the chiral symmetry breaking dynamics. For simplicity, in what follows we will assume that the gauge couplings $g_{i j}=g$ are the same for every site except for $(1,1)$. The rules of naive dimensional analysis [10] then imply that the scale $\Lambda$ of the underlying high-energy dynamics which gives rise to this theory is bounded by of order $4 \pi f$. 


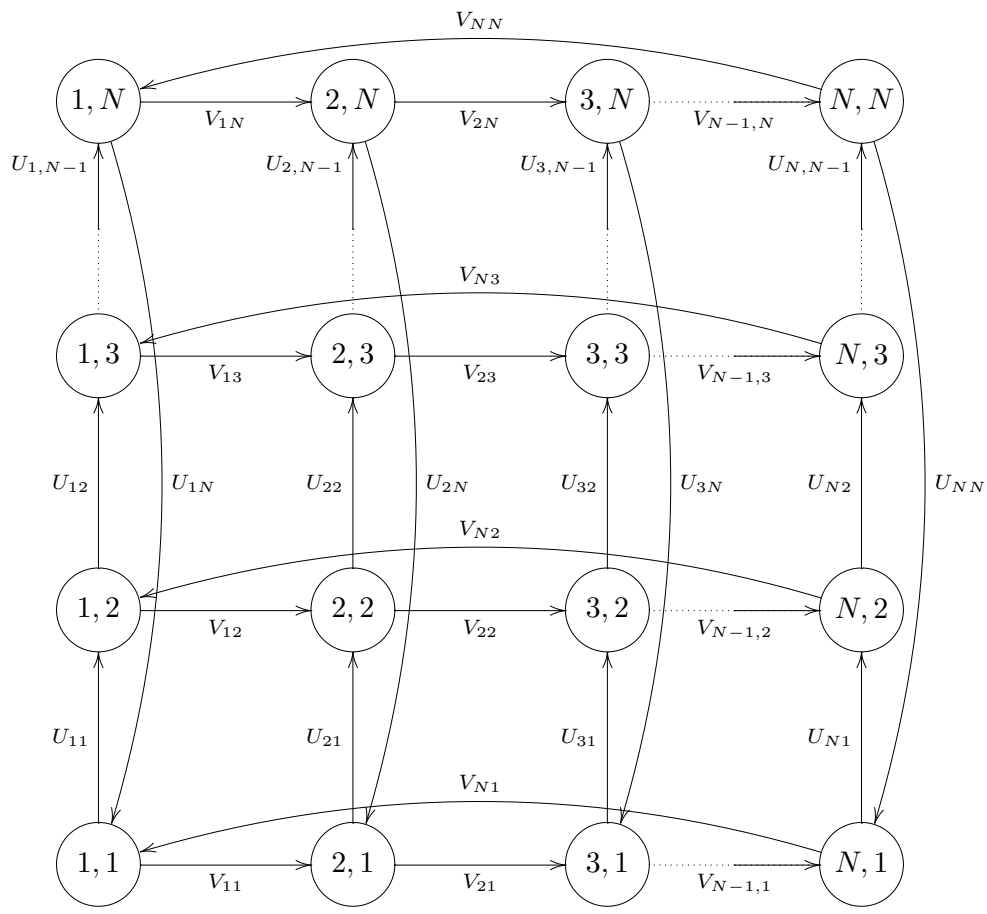

Figure 1: A composite Higgs model based on an $N \times N$ toroidal lattice "theory space." $S U(3)$ gauge groups live at every site except $(1,1)$, while the links represent non-linear sigma fields transforming as $(3, \overline{3})$ 's under the adjacent gauge symmetries. Only an $S U(2) \times U(1)$ subgroup of an $S U(3)$ global symmetry group is gauged at site $(1,1)$. As described in the text, $N^{2}-1$ sets of Goldstone bosons are eaten, $N^{2}-1$ get mass from "plaquette operators" which explicitly break the chiral symmetries, and two sets remain in the very low-energy theory. This illustration comes from [9].

The $2 N^{2}$ Goldstone bosons of the chiral symmetry breaking dynamics are incorporated into the sigma-model fields

$$
U_{i j}=\exp 2 i \pi_{u, i j} / f, \quad V_{i j}=\exp 2 i \pi_{v, i j} / f .
$$

The gauge symmetry breaking pattern implied is $S U(3)^{N^{2}-1} \times S U(2) \times U(1) \rightarrow$ $S U(2) \times U(1)$, resulting in $N^{2}-1$ sets of "eaten" Goldstone bosons. The remaining $N^{2}+1$ sets of Goldstone bosons in the physical spectrum interact via the gauge interactions, which explicitly violate the chiral symmetries. However, because of the "topology" of theory space, the lowest-order interaction in the effective theory which breaks the chiral-symmetries in the same way as the gauge interactions only occurs at high order [1]. Therefore, the leading contribution to the masses of these remaining scalars from the low-energy gauge interactions is finite, and arises at $\mathcal{O}\left(g^{4}\right)$ from the Coleman-Weinberg potential [15] . 
An important ingredient in these models is a set of nonderivative chiral-symmetry breaking operators of the form of "plaquette" interactions 3

$$
\mathcal{L}_{p l}=\lambda f^{4} \sum_{i j} \operatorname{Tr}\left(U_{i j} V_{i j+1} U_{i+1}^{\dagger}{ }_{j} V_{i j}^{\dagger}\right)+\text { h.c. }
$$

where (again, for simplicity) we have assumed that the dimensionless coupling constants $\lambda$ are the same for every plaquette. Expanding these operators in terms of the Goldstone bosons fields, we find

$$
\mathcal{L}_{p l}=-4 \lambda f^{2} \sum_{i j} \operatorname{Tr}\left(\pi_{u, i j}+\pi_{v, i j+1}-\pi_{u, i+1} j-\pi_{v, i j}\right)^{2}+\mathcal{O}\left(\pi^{4}\right)+\ldots
$$

These operators have the extraordinary feature that they give rise to masses to $N^{2}-1$ of the remaining scalars, but leave massless the two combinations

$$
\pi_{u, i j} \equiv \frac{U}{N} \quad \pi_{v, i j} \equiv \frac{V}{N}
$$

which are uniform in either the "u" or "v" directions. The factors of $N$ arise so as to normalize the $U$ and $V$ fields correctly. Both the $U$ and $V$ fields contain $S U(2) \times U(1)$ doublet scalars $\phi_{u}$ and $\phi_{v}$ with the quantum numbers of the Higgs boson. The theory gives rise to two light (so far in this discussion, massless) composite Higgs bosons with nonderivative interaction of the form [1, 2]

$$
\mathcal{L}_{p l} \supset \frac{4 \lambda}{N^{2}} \operatorname{Tr}\left(\phi_{u} \phi_{u}^{\dagger}-\phi_{v} \phi_{v}^{\dagger}\right)^{2}+\frac{4 \lambda}{N^{2}}\left(\phi_{u}^{\dagger} \phi_{u}-\phi_{v}^{\dagger} \phi_{v}\right)^{2} .
$$

Additionally, a negative mass-squared for one or both Higgs bosons may be introduced either through a symmetry-breaking plaquette operator at the site $(1,1)$ [1] or through the effect of coupling the Higgs bosons to the top-quark [2]. In either case, the resulting mass-squared of the Higgs is of order

$$
\left|m_{h}\right|^{2} \simeq \frac{\lambda v^{2}}{N^{2}} .
$$

The left- and right-handed quarks and leptons transform under the $S U(2) \times U(1)$ gauge interactions at the site $(1,1)[1$, 2]. For the light fermions, Yukawa couplings between the fermions and the composite Higgs bosons are introduced. Such interactions violate the chiral symmetries protecting the Higgs bosons masses, but the size of the resulting corrections is small since $m_{q} \ll v$. This choice preserves a $(U(2))^{5}$ flavor symmetry, broken only by the Yukawa couplings to the composite Higgs, suppressing flavor-changing neutral currents from the $S U(2) \times U(1)$ and $S U(3)^{N^{2}-1}$ gauge bosons. Because the light quarks obtain mass from Yukawa couplings to the

\footnotetext{
${ }^{3}$ Because of the reduced symmetry at site $(1,1)$, additional operators are present there which play an important role in the detailed phenomenology of the composite scalar particles. [1, 2]
} 
composite scalars, the bounds on the compositeness scale derived in Section apply to this model. As noted above, however, the light Higgs boson is "delocalized" in theory space, eqn. (19), and therefore has only an amplitude of order $1 / N$ of being at site $(1,1)$. Consequently, we would say that $\Lambda$ must satisfy

$$
\Lambda \gtrsim 21 \mathrm{TeV} \sqrt{\kappa N\left(\frac{m_{c}}{1.5 \mathrm{GeV}}\right)},
$$

and be at least of order $\sqrt{N} \cdot 75 \mathrm{TeV}$ for $\kappa=4 \pi$.

The top-quark presents a more difficult problem. In this case, no direct Yukawa coupling is introduced [1]. Instead, the top-quark is "spread out" in theory space: a family of massive $S U(3)$ vector fermions on the sites $\left(1, n_{v}\right)$ and $\left(n_{u}, 1\right)$ is added (here $1 \leq n_{u, v} \leq N$ ), along with local interactions between the the vector fermions at adjacent sites and the gauge-eigenstate top-quark (which has $S U(2) \times U(1)$ gauge interactions at site $(1,1))[1$, 2]. Upon diagonalizing the resulting mass matrix, the expected order-one Yukawa coupling, $y_{t}$, of the Higgs to the top-quark is generated so long as the nearest neighbor couplings are of order one and the lightest vectorfermion mass $m$ satisfies $m / f \simeq y_{t}$.

One might imagine that the bounds of Section 2 could be evaded in a different class of models in which the light fermions are also spread out in theory space, perhaps with "families" of $S U(3)$ vector fermions. Even in this case, however, the crucial flavor-violating couplings are still Yukawa couplings between an ordinary fermion at the site $(1,1)$ and the appropriate component of a vector fermion at an adjacent site. The bounds described in Section 2 apply to these couplings and constrain the corresponding models.

\section{Flavor and Fine-Tuning in Theory Space}

In order to understand the implications of the lower bound from flavor physics, we will examine an upper bound imposed by the wish to avoid fine-tuning in the Higgs boson masses. As noted before, the chiral symmetries of theory space imply that the leading contributions to the Higgs boson masses are finite contributions arising from the Coleman-Weinberg potential. The rules of power-counting are easily modified in this case [1,2] in order to estimate the size of these finite contributions to parameters in the low-energy theory. In particular, the size of these contribution is the same as that in a standard scalar Higgs model with a cutoff equal to the mass of the lowest appropriate resonance.

For example, gauge boson loop corrections to the Higgs boson masses are of order [1, 2]

$$
\delta m_{H}^{2} \simeq \frac{e^{2}}{16 \pi^{2} \sin ^{2} \theta_{W}}\left(\frac{g f}{N}\right)^{2} \simeq\left(\frac{\alpha}{4 \pi \sin ^{2} \theta_{W}}\right)^{2} \Lambda^{2},
$$

where the mass of the first vector resonance (of order $g f / N$ ) plays the role of the cutoff of the low-energy theory, and we have assumed that $g=\mathcal{O}\left(\mathrm{Ne} / \sin \theta_{W}\right)$ in 
order to yield the appropriate low-energy weak coupling constant. Here, and in the rest of this paper, we take $\Lambda \simeq 4 \pi f$ which corresponds to $\kappa=4 \pi$ above. The size of these finite corrections to the Higgs boson mass must be compared to the desired low-energy mass-squared given in eqn. (21). To avoid fine-tuning, we require that

$$
\left|\frac{\delta m_{H}^{2}}{m_{H}^{2}}\right| \lesssim 1,
$$

which yields

$$
\Lambda \lesssim\left(\frac{4 \pi \sin ^{2} \theta_{W}}{\alpha}\right) \frac{\sqrt{\lambda} v}{N} \approx \frac{108 \mathrm{TeV} \sqrt{\lambda}}{N}
$$

If gauge-boson loop corrections were the only issue, the cutoff could be taken to be of order $100 \mathrm{TeV}$ without any fine-tuning.

However, the most important corrections to the Higgs boson masses arise from the interactions added to give rise to the top-quark mass. The fermion loop ColemanWeinberg contribution to the Higgs mass-squared is of order

$$
\left|\delta m_{H}^{2}\right| \simeq \frac{N_{c} y_{t}^{2} m^{2}}{16 \pi^{2}} \approx \frac{N_{c} y_{t}^{4}}{\left(16 \pi^{2}\right)^{2}} \Lambda^{2}
$$

where $N_{c}=3$ accounts for color. In this case, the absence of fine-tuning $\left(\delta m_{H}^{2} / m_{H}^{2} \lesssim\right.$ 1) implies

$$
\Lambda \lesssim \frac{16 \pi^{2} \sqrt{\lambda} v}{\sqrt{N_{c}} y_{t}^{2} N} \approx \frac{22 \mathrm{TeV} \sqrt{\lambda}}{N} .
$$

Comparing eqns. (27) and (22) we see that for $N=2$ fine-tuning on the order of $1 \%$ is required if the bound from $\Delta C=2$ mixing is to be satisfied. If the bound from $\mathrm{CP}$ violation (13) must also be satisfied, the fine-tuning required is of order $.04 \%$.

\section{Isospin Violation}

A crucial issue in all composite Higgs models is the size of weak-isospin violation [16, 8, 17, 18]. Recall that the standard one-doublet Higgs model has an accidental custodial isospin symmetry [19], which naturally implies that the weak-interaction $\rho$-parameter is approximately one. While all $S U(2) \times U(1)$ invariant operators made of a single scalar-doublet field that have dimension less than or equal to four automatically respect custodial symmetry, terms of higher dimension that arise from the underlying physics at scale $\Lambda$ in general will not. Furthermore, the interaction given in eqn. (20) does not respect custodial symmetry. However, the effect of these interactions is to introduce custodial violation in the spectrum of Higgs boson masses and therefore only affects the weak interaction $\rho$ parameter at one-loop. 
The embedding of $S U(2) \times U(1)$ in a global $S U(3)$ interaction is identical to the symmetry structure of the "Banks model", which is known to give rise to isospin violation [3]. This violation is most directly understood by expanding the kinetic energy terms in eqn. (15) to fourth-order in the pion fields. Keeping only the terms involving $\phi_{u}$ and $\phi_{v}$, we find the isospin violating interactions

$$
\mathcal{L}_{k i n} \supset-\frac{1}{6 N f^{2}}\left[\left(\partial_{\mu} \phi_{u}^{\dagger} \phi_{u}\right)^{2}-\left(\partial_{\mu} \phi_{u}^{\dagger} \phi_{u}\right)\left(\phi_{u}^{\dagger} \partial^{\mu} \phi_{u}\right)+\left(\phi_{u}^{\dagger} \partial^{\mu} \phi_{u}\right)^{2}\right]+u \leftrightarrow v .
$$

Writing the vevs of the Higgs fields as

$$
\left\langle\phi_{u}\right\rangle=\left(\begin{array}{c}
0 \\
\frac{v \cos \beta}{\sqrt{2}}
\end{array}\right) \quad\left\langle\phi_{v}\right\rangle=\left(\begin{array}{c}
0 \\
\frac{v \sin \beta}{\sqrt{2}}
\end{array}\right),
$$

we find the contribution

$$
\Delta \rho^{\star}=\alpha \Delta T=\frac{v^{2}}{4 N^{2} f^{2}}\left(1-\frac{\sin ^{2} 2 \beta}{2}\right) .
$$

Current limits derived from precision electroweak observables [18] require that $\Delta T \lesssim$ 0.5 at $95 \%$ confidence level for a Higgs mass less than $500 \mathrm{GeV}$. The bound in eqn. 30 implies that

$$
\Lambda \simeq 4 \pi f \gtrsim \frac{25 \mathrm{TeV}}{N}\left(1-\frac{\sin ^{2} 2 \beta}{2}\right)^{1 / 2} .
$$

Comparing this with eqn.(27), we see that the underlying strong dynamics cannot be at energies much less than $10 \mathrm{TeV}$, even if the high-energy theory contains approximate flavor and CP symmetries that nullifies the limits of (6) and (13).

\section{Discussion}

In this paper, we have shown that the size of flavor violating interactions arising generically from underlying flavor dynamics in composite Higgs models constrain the compositeness scale to be at least $75 \mathrm{TeV}$. This bound applies not only to the original composite higgs models [3], but also to the recently developed "theory space" models [1, 2]. For theory space models based on an $N \times N$ toroidal lattice, the lower limit is $\Lambda \gtrsim 75 \mathrm{TeV} \sqrt{N}$, so that the bound is $105 \mathrm{TeV}$ for $N=2$. On the other hand, if fine-tuning of the higgs mass is to be avoided in such models, $\Lambda \lesssim 22 \operatorname{TeV} \sqrt{\lambda} / N$; preventing flavor-changing neutral currents then leads to fine-tuning at the level of $10 / N^{3} \%$. We have also seen that the lower limit on $\Lambda$ derived from considering weak isospin violation are somewhat weaker than those from FCNC, while those from CP-violation in the neutral Kaon system are potentially much stronger.

It is also interesting to note how one might construct models that are not constrained by the bounds discussed in this paper. In order to produce the appropriate 
Yukawa couplings without potentially large effects in neutral-meson mixing, the underlying flavor or strong dynamics must incorporate additional structure. First, it may be possible to construct a theory in which the charm mass-eigenstates are eigenstates of the corresponding flavor gauge-interactions. In this case, no $\Delta C=2$ interactions arise at the scale relevant for producing the charm-quark yukawa couplings. Since Cabibbo mixing exists, however, such interactions will necessarily arise at the scale relevant for strange-quark mass generation, yielding the result of eqn. (11). Second, the underlying strong dynamics could potentially be arranged to have a different scaling behavior, analogous to "walking technicolor" [20]. In this case one might have Yukawa couplings of order $\Lambda / M$ rather than the square of that ratio. Or third, the underlying flavor dynamics could incorporate an approximate GIM symmetry [21, 22]. Similarly, if the underlying dynamical theory incorporated an approximate CP symmetry, then the low-energy theory would not necessarily make the dangerously large contributions to $\varepsilon$ discussed here.

In summary, we have seen that the low-energy structure of the composite Higgs model alone is not sufficient to eliminate potential problems with flavor-changing neutral current or excessive $\mathrm{CP}$ violation; solving those problems requires additional information or assumptions about the symmetries of the underlying strong dynamics.

Note Added: After the completion of this manuscript, two minimal composite Higgs models have recently been proposed [23, 24]. As noted by those authors, the constraints discussed in this note are relevant to the new models as well.

\section{Acknowledgments}

We thank Andrew Cohen, Nima Arkani-Hamed, Hong-Jian He, Ken Lane, and Martin Schmaltz for helpful discussions. This work was supported in part by the Department of Energy under grant DE-FG02-91ER40676 and by the National Science Foundation under grant PHY-0074274.

\section{References}

[1] N. Arkani-Hamed, A. G. Cohen and H. Georgi, Phys. Lett. B 513 (2001) 232 arXiv:hep-ph/0105239.

[2] N. Arkani-Hamed, A. G. Cohen, T. Gregoire and J. G. Wacker, arXiv:hep$\mathrm{ph} / 0202089$.

[3] D. B. Kaplan and H. Georgi, Phys. Lett. B136 (1984) 183; Phys. Lett. B145 (1984) 216 ;

T. Banks, Nucl. Phys. B 243 (1984) 123;

D. B. Kaplan, H. Georgi, and S. Dimopoulos, Phys. Lett. B136 (1984) 187;

M. J. Dugan, H. Georgi, and D. B. Kaplan, Nucl. Phys. B 254 (1985) 299. 
[4] N. Arkani-Hamed, A. G. Cohen and H. Georgi, Phys. Rev. Lett. 86 (2001) 4757 arXiv:hep-th/0104005.

[5] H. C. Cheng, C. T. Hill and J. Wang, Phys. Rev. D 64 (2001) 095003 arXiv:hep-ph/0105323.

[6] S. Dimopoulos and L. Susskind, Nucl. Phys. B155 (1979) 237.

[7] E. Eichten and K. Lane, Phys. Lett. B90 (1980) 125.

[8] R. S. Chivukula, B. A. Dobrescu and E. H. Simmons, Phys. Lett. B 401 (1997) 74 arXiv:hep-ph/9702416.

[9] K. Lane, arXiv:hep-ph/0202093.

[10] A. Manohar and H. Georgi, Nucl. Phys. B234 (1984) 189; H. Georgi and L. Randall, Nucl. Phys. B276 (1986) 241; T. Appelquist, Scottish Summer School (1980) 385.

[11] H. Georgi, Phys. Lett. B298 (1993) 187, hep-ph/9207278.

[12] D.E. Groom et al, The European Physical Journal C15 (2000) 1, and 2001 off-year partial update for the 2002 edition available on the PDG WWW pages (URL: http://pdg.lbl.gov/)

[13] See, for example, A. Ali Khan et al. [CP-PACS Collaboration], Phys. Rev. D 64, 034505 (2001) arXiv:hep-lat/0010009.

[14] H. Georgi, Nucl. Phys. B 266, 274 (1986); M. R. Douglas and G. W. Moore, arXiv:hep-th/9603167.

[15] S. R. Coleman and E. Weinberg, Phys. Rev. D 7 (1973) 1888.

[16] R. S. Chivukula and E. H. Simmons, Phys. Lett. B 388 (1996) 788 arXiv:hep$\mathrm{ph} / 9608320$.

[17] R. S. Chivukula, E. H. Simmons and B. A. Dobrescu, arXiv:hep-ph/9703206.

[18] R. S. Chivukula and N. Evans, Phys. Lett. B 464 (1999) 244 arXiv:hepph/9907414. ; R. S. Chivukula, C. Hoelbling and N. Evans, Phys. Rev. Lett. 85 (2000) 511 arXiv:hep-ph/0002022. ; R. S. Chivukula, arXiv:hep-ph/0005168. ; R. S. Chivukula and C. Hoelbling, in Proc. of the APS/DPF/DPB Summer Study on the Future of Particle Physics (Snowmass 2001) ed. R. Davidson and C. Quigg, arXiv:hep-ph/0110214.

[19] S. Weinberg, Phys. Rev. D19 (1979) 1277; L. Susskind, Phys. Rev. D20 (1979) 2619; P. Sikivie, et. al., Nucl. Phys. B173 (1980) 189. 
[20] B. Holdom, Phys. Lett. B105 (1985) 301; K. Yamawaki, M. Bando and K. Matumoto, Phys. Rev. Lett. 56 (1986) 1335; V.A. Miransky, Nuovo Cim. 90A (1985); T. Appelquist, D. Karabali, and L.C.R. Wijewardhana, Phys. Rev. D35(1987) 389; 149; T. Appelquist and L.C.R Wijewardhana, Phys. Rev. D35 (1987) 774; Phys. Rev. D36 (1987) 568.

[21] S. Dimopoulos, H. Georgi, and S. Raby, Phys. Lett. B127 (1983) 101;

S.-C. Chao and K. Lane, Phys. Lett. B159 (1985) 135; L. Randall Nucl.Phys. B403 (1993) 122, hep-ph/9210231.

[22] R. S. Chivukula and H. Georgi, Phys.Lett. 188B (1987) 99 and Phys.Rev. D36 (1987) 2102; R. S. Chivukula, H. Georgi, and L. Randall, Nucl. Phys. B292 (1987) 93.

[23] N. Arkani-Hamed, A. G. Cohen, E. Katz and A. E. Nelson, arXiv:hep$\mathrm{ph} / 0206021$.

[24] N. Arkani-Hamed, A. G. Cohen, E. Katz, A. E. Nelson, T. Gregoire and J. G. Wacker, arXiv:hep-ph/0206020. 